\title{
Article \\ A TRICk to Improve the Effectiveness of RIC: Role of Limb Temperature in Enhancing the Effectiveness of Remote Ischemic Conditioning
}

\author{
Claudia Penna ${ }^{1}$, Matteo Sorge ${ }^{2} \mathbb{D}$, Francesca Tullio ${ }^{1}$, Stefano Comità ${ }^{1}$, Saveria Femminò ${ }^{3} \mathbb{D}$, Mara Brancaccio $^{2, *} \mathbb{D}$ \\ and Pasquale Pagliaro ${ }^{1, * \mathbb{D}}$ \\ 1 Department of Clinical and Biological Sciences, University of Turin, 10043 Orbassano, Italy; \\ claudia.penna@unito.it (C.P.); francesca.tullio@unito.it (F.T.); stefano.comita@unito.it (S.C.) \\ 2 Department of Molecular Biotechnology and Health Sciences, University of Turin, 10126 Turin, Italy; \\ matteo.sorge@unito.it \\ 3 Department of Medical Sciences, University of Turin, 10126 Turin, Italy; saveria.femmino@unito.it \\ * Correspondence: mara.brancaccio@unito.it (M.B.); pasquale.pagliaro@unito.it (P.P.)
}

check for

updates

Citation: Penna, C.; Sorge, M.; Tullio,

F.; Comità, S.; Femminò, S.;

Brancaccio, M.; Pagliaro, P. A TRICk to Improve the Effectiveness of RIC:

Role of Limb Temperature in

Enhancing the Effectiveness of

Remote Ischemic Conditioning.

Biology 2022, 11, 146. https://

doi.org/10.3390/biology11010146

Academic Editor: Mridula Chopra

Received: 2 December 2021

Accepted: 14 January 2022

Published: 17 January 2022

Publisher's Note: MDPI stays neutral with regard to jurisdictional claims in published maps and institutional affiliations.

Copyright: (c) 2022 by the authors. Licensee MDPI, Basel, Switzerland. This article is an open access article distributed under the terms and conditions of the Creative Commons Attribution (CC BY) license (https:// creativecommons.org/licenses/by/ $4.0 /)$.
Simple Summary: Remote ischemic conditioning is a simple cardioprotective practice consisting in brief intermittent ischemia applied to a limb. Remote ischemic conditioning has been repeatedly validated in animal models. However, translation from animal experiments to clinics for remote ischemic conditioning has been disappointing. We have demonstrated that keeping the animal's limb warm while performing intermittent ischemia reduces infarct size more effectively than cold intermittent ischemia; thus, we propose that a more accurate temperature control of the limb undergoing remote ischemic conditioning can increase the efficacy of this cardioprotective maneuver. A simple thermal blanket around the ischemic limb while performing remote ischemic conditioning could be an easy approach to test in humans, as it is simple and safe.

Abstract: Background: Treatment of myocardial ischemia/reperfusion (IR) injury is still an unmet clinical need. A large variability of remote ischemic conditioning (RIC) protection has been reported; however, no studies have considered the temperature of the ischemic limb. We analyzed the effects of temperature on RIC protection. Methods: Left hind-limbs of anesthetized male mice were immersed in warm $\left(40^{\circ} \mathrm{C}\right.$, warm-RIC) or cold $\left(20^{\circ} \mathrm{C}\right.$, cold-RIC) water and subjected to a RIC protocol $(4 \times 5 \mathrm{~min}$ limb ischemia/reperfusion). In the control groups (warm-CTR or cold-CTR), the limbs underwent thermic conditions only. Isolated hearts underwent $30 \mathrm{~min}$ ischemia and $60 \mathrm{~min}$ reperfusion. A PI3K-inhibitor, LY294002 (5 $\mu \mathrm{M}$ ), was infused in warm-RIC hearts before the IR protocol (warm-RIC LY). Infarct size was evaluated by nitro blue tetrazolium staining and expressed as the percent of risk area. Results: While cold-RIC did not reduce the infarct size compared to cold-CTR (51 $\pm 1.62 \%$ vs. $54 \pm 1.07 \%$ of risk area, $p=$ NS), warm-RIC ( $44 \pm 1.13 \%)$ significantly reduced the infarct size with respect to either cold-RIC ( $p<0.001)$ or warm-CTR $(58 \pm 1.41 \%, p<0.0001)$. LY294002 infusion revealed the PI3K/Akt involvement in the warm-RIC protection. Infarct size reduction was abrogated by LY294002 pretreatment (warm-RIC: $44 \pm 1.13 \%$ vs. warm-CTR $58 \pm 1.41 \% p<0.0001$; vs. warm-RIC LY $54 \pm 1.69 \% p=0.0002$ ). Conclusion: our study shows a remarkable difference between warm-RIC and cold-RIC in terms of infarct size reduction, supporting a pivotal role for limb temperature in RIC-induced cardioprotection.

Keywords: cardioprotection; remote ischemic conditioning; infarct size; reperfusion injury

\section{Introduction}

Despite the successes achieved against cardiovascular diseases, the prevalence of these diseases, including heart failure, continues to rise. In particular, ischemic disease 
is the leading cause of heart failure [1]; therefore, understanding the mechanisms of ischemia/reperfusion injury and finding new cardioprotective mechanisms is of paramount importance.

Currently, the only way to salvage ischemic myocardium from infarction and to limit infarct size is timely reperfusion. However, reperfusion per se adds an irreversible component of damage to the myocardium and thus contributes to infarct size (namely, ischemia/reperfusion injury, IRI) [2-4]. Despite improvements in the therapy of acute myocardial infarction over recent years, the incidence of myocardial infarction in the aged population is not declining and the number of survivors from acute myocardial infarction that go on to develop heart failure is increasing [5]. Therefore, treatment of IRI is still a major unmet clinical need and additional cardioprotective strategies are required [6,7].

Brief non-lethal episodes of myocardial ischemia and reperfusion protect the myocardium from the consequences of IRI by activating intrinsic molecular mechanisms [2-4]. Such protection by ischemic conditioning can be induced locally in the heart and in tissues remote from the protected organ — that is, so-called remote ischemic conditioning (RIC). RIC can be induced prior (remote preconditioning or pre-RIC), during (remote percoditioning or per-RIC) or after (remote postconditioning or post-RIC) a prolonged myocardial ischemia [2-4]. RIC induces the activation of the reperfusion injury salvage kinase (RISK) pathway (including PI3K/Akt pathway) and/or the survivor activating factor enhancement (SAFE) pathway within the heart, with some species differences in the involvement of both pathways [2-4].

Although evidence for the efficacy of RIC cardioprotective maneuvers has been described in all species tested so far, including humans [2-4], translation from animal experiments to clinical practice remains challenging and has been disappointing [8]. Additionally, large-scale randomized controlled trials have failed to confirm RIC-induced cardioprotection in patients [9]. A great variability in RIC protection among different laboratories has been described $[10,11]$ and it is likely that the variability is greater than the actual perception of the phenomenon. Indeed, we should consider that RIC maneuvers lack standardization in terms of the number and duration of the brief cycles of ischemia and reperfusion, and that this may be one of the reasons for the variability in results.

Nevertheless, despite similarities in terms of number and duration, results can be still different in terms of efficacy of IRI limitation. Actually, in their interesting systematic review and meta-analysis, Bromage et al. reported a significant heterogeneity in effect size that could not be explained by any of the experimental variables analyzed by metaregression [10]. From this analysis, it emerged that studies lack consistency in quality and study design. Indeed, other parameters can impact RIC maneuver efficacy. Intriguingly, to the best of our knowledge, no studies have considered the temperature of the ischemic limb among the variables to be controlled (limb temperature was not mentioned in the meta-analysis by Bromage et al. [10]). There is, therefore, a clear need for better-performed studies, with a particular emphasis on the detailed characterization of RIC protocols and investigation of the potential impact of limb temperature.

To this aim, we used a standard pre-RIC protocol performed at two controlled limb temperatures, a cold temperature $\left(20^{\circ} \mathrm{C}\right.$; cold-RIC) and a warm temperature $\left(40^{\circ} \mathrm{C}\right.$; warmRIC), to compare the cardioprotective efficacy of these two protocols in a model of cardiac ischemia/reperfusion. Here we demonstrate that it is enough to keep the ischemic conditioned limb warm $\left(40^{\circ} \mathrm{C}\right)$ to increase the cardioprotective efficacy of this practice, and that protection is causally due to PI3K/Akt activation within the myocardium.

\section{Materials and Methods}

\subsection{Animals}

Male FVB mice ( $n$ total 60 ) received humane care in compliance with Italian law (DL116, 27 January 1992) and in accordance with the Guide for the Care and Use of Laboratory Animals published by the US National Institute of Health (NIH Publication No. 85-23, revised 1996). All efforts were made to minimize suffering. The local "Animal Use and 
Care Committee" approved the animal protocol (protocol no: E669C.44). The mouse is an animal species quite routinely used in the cardioprotection field [11], and in particular in RIC studies [10].

\subsection{Remote Conditioning Protocol}

Male FVB mice weighing between 25 and 35 g (10-15 weeks old) were given $500 \mathrm{U}$ heparin and anesthetized with sodium pentothal $(50 \mathrm{mg} / \mathrm{kg})$ by intraperitoneal injections before being subjected to the Remote Ischemic Conditioning (RIC) protocol.

Anesthetized animals had a small cuff placed around their left hind limb and were randomized to receive either the (A) Control protocol, consisting of a time period (45 $\mathrm{min}$ ) with no cuff inflation or (B) RIC protocol, consisting of 4 cycles of 5 min cuff inflation to $200 \mathrm{mmHg}$ with intermittent 5 min deflations [12]. The experiments in group A and B were performed with the studied limb immersed in water, whose temperature was kept at either 20 or $40{ }^{\circ} \mathrm{C}$ (Figure $\left.1 \mathrm{~A}\right)$.

A

In vivo procedure

Control

RIC

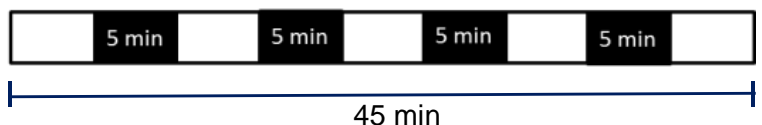

B

Ex vivo procedure

Ischemia

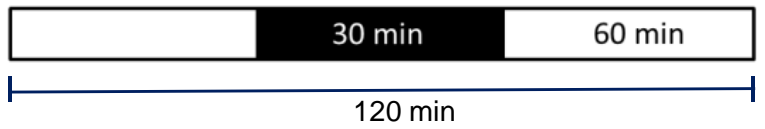

Figure 1. Experimental protocol. (Panel A): anesthetized mice underwent thermic procedures in which the left hindlimb was immersed in cold $\left(20^{\circ} \mathrm{C}\right)$ or warm water $\left(40^{\circ} \mathrm{C}\right)$ only (Controls) or underwent a remote conditioning (RIC) protocol while the hindlimb was immersed in water. (Panel B): isolated mice hearts underwent a global normothermic $\left(37^{\circ} \mathrm{C}\right)$ ischemia/reperfusion procedure. Black boxes represent periods of ischemia; white boxes represent periods of normal perfusion.

Therefore, the experimental groups were:

1. Cold-Controls (cold-CTR): left hind limb was maintained at a temperature of $20^{\circ} \mathrm{C}$ for 45 min without cuff inflation;

2. Warm-Controls (warm-CTR): left hind limb was maintained at a temperature of $40{ }^{\circ} \mathrm{C}$ for 45 min without cuff inflation;

3. Cold-RIC (cold-RIC): RIC was performed while the left hind limb was maintained at a temperature of $20^{\circ} \mathrm{C}$;

4. Warm-RIC (warm-RIC): RIC was performed while the left hind limb was maintained at a temperature of $40{ }^{\circ} \mathrm{C}$.

At the end of the RIC protocol, the animals were sacrificed and the hearts were excised and perfused on a Langendorff apparatus, using the method described below.

\subsection{Isolated Heart Perfusion Technique}

The animals, while still anesthetized, were culled by cervical dislocation [13]. Hearts were rapidly excised and perfused retrogradely at $80 \mathrm{mmHg}$ by the Langendorff technique with Krebs-Henseleit bicarbonate buffer containing (mM) $\mathrm{NaCl} 118, \mathrm{NaHCO}_{3} 25, \mathrm{KCl}$ 4.7, $\mathrm{KH}_{2} \mathrm{PO}_{4} 1.2, \mathrm{MgSO}_{4} 1.2, \mathrm{CaCl}_{2} 1.25$ and Glucose 11 . The buffer was gassed with $95 \% \mathrm{O}_{2}$ : 
$5 \% \mathrm{CO}_{2}$. The temperature of the perfusion system was maintained at $37^{\circ} \mathrm{C}$. After $30 \mathrm{~min}$ of stabilization, 9 hearts were not used as they did not start to beat properly.

The isolated hearts, after stabilization, were subjected to $30 \mathrm{~min}$ of global ischemia followed by 60 min of reperfusion (IR) at $37^{\circ} \mathrm{C}$ (Figure 1B) [14]. A subgroup of warmRIC hearts was also treated with $5 \mu \mathrm{M}$ of the PI3K inhibitor LY294002 (warm-RIC LY) for 10 min before global ischemia. The dose of LY294002 was chosen on the basis of previous experiments [15].

The isolated heart allowed us to perform the IR protocol at well controlled temperatures to avoid influences on infarct size due to differences in body temperature [16]. Therefore, the groups differed only in ischemic limb temperature before undergoing heart isolation.

At the end of the perfusion period, hearts were removed from the perfusion apparatus and used for infarct size assessment [14].

\subsection{Infarct Size Assessment}

In brief, infarct areas were assessed with the nitro blue tetrazolium (NBT) technique in a blinded fashion, as previously described [14]. Immediately after reperfusion, hearts were removed from the perfusion apparatus and the ventricles were dissected by transverse sections into 2-3 slices. Following $20 \mathrm{~min}$ of incubation at $37{ }^{\circ} \mathrm{C}$ in $0.1 \%$ solution NBT (Sigma-Aldrich, St. Louis, MO, USA) in a phosphate buffer, unstained necrotic tissue was carefully separated from stained viable tissue by an independent observer, who was unaware of the protocols. The measurement of infarct size was performed first by planimetry, then immediately after by weighing the separated tissue by two different operators. Since a good correlation was found between the two measurements, we decided to report the data obtained by weighing, as they represented the mass of necrotic tissue more accurately. Since the ischemia was global and since we analyzed only the ventricles, the necrotic mass was expressed as a percentage of the analyzed ischemic tissue.

The apical slice of the ventricles, due to its small size, was not used for infarct size assessment, but was immediately frozen and subsequently analyzed by Western blot analysis [14].

\subsection{Western Blotting}

Frozen samples were powdered in liquid nitrogen and lysed in Tris-buffered saline with 1\% Triton X-100, plus Roche complete protease inhibitor cocktail, NaF $10 \mathrm{mM}$, PMSF $1 \mathrm{mM}$ and Na3VO4 $1 \mathrm{mM}$. Protein extracts were clarified by centrifugation for $20 \mathrm{~min}$ at $20,000 \times g$ at $4{ }^{\circ} \mathrm{C}[14]$.

Western blot band quantifications were performed with Image Lab software (Bio-Rad). Antibodies against Akt used: Akt (1:1000, \#4691 Cell Signaling), P-Thr308 Akt (1:1000, \#4056 Cell Signaling). As the secondary antibody: Anti-rabbit IgG-peroxidase antibody produced in goat (1:5000, \#A6154, Sigma-Aldrich).

\subsection{Statistical Analysis}

All values are expressed as mean \pm SE. Data were analyzed using one or two-way analysis of variance (ANOVA) followed by the Bonferroni post hoc test. For all analyses, a minimum value of $p<0.05$ was considered significant. Statistical analyses were performed using GraphPad Prism 4 (GraphPad Software version 4.0).

\section{Results}

\subsection{Warm-RIC Reduced the Extent of Myocardial Infarction More Effectively Than Cold-RIC}

In both cold- or warm-CTR groups, the infarct size after $30 \mathrm{~min}$ ischemia and $60 \mathrm{~min}$ of reperfusion were $54 \pm 1.07 \%$ and $58 \pm 1.41 \%$ of risk zone $(p=$ Not Significant (NS)), respectively. The cold-RIC did not significantly reduce the infarct size $(51 \pm 1.62 \%$ of risk zone, NS with respect to cold-CTR), while the warm-RIC significantly reduced the infarct 
size $(44 \pm 1.13 \%, p<0.0001$ with respect to warm-CTR; $p<0.001$ with respect to cold-RIC; Figure 2A).
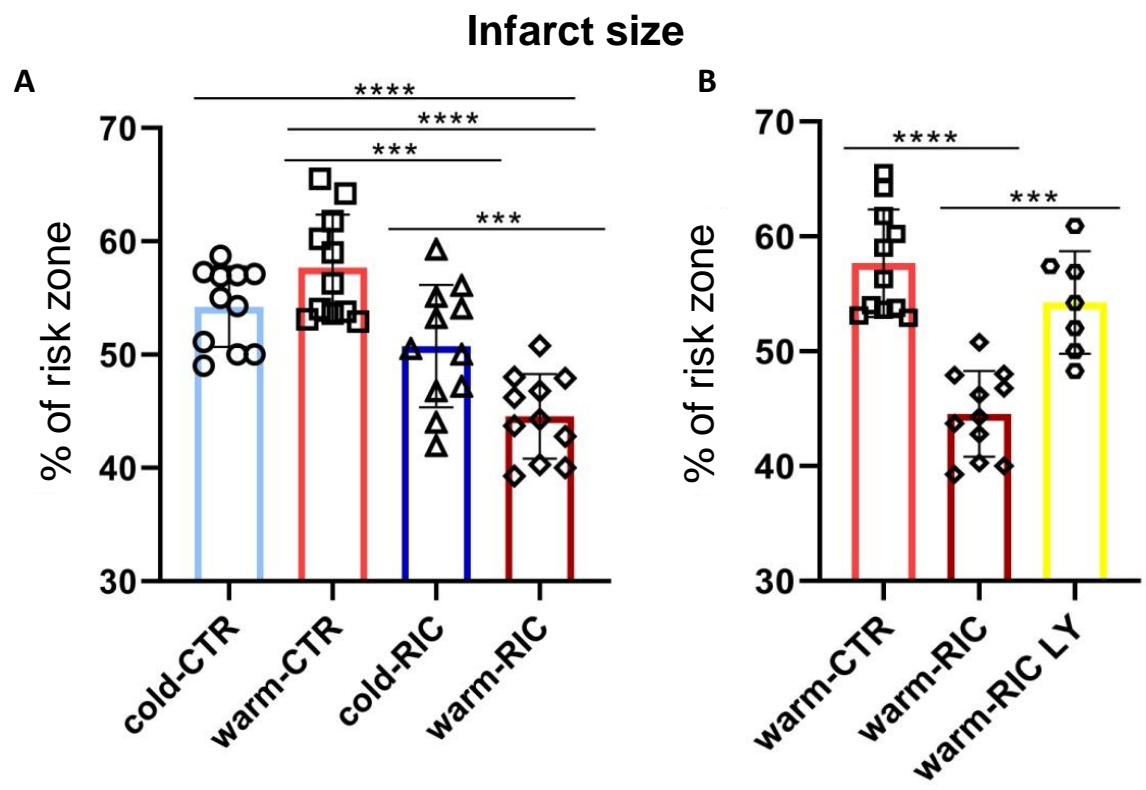

Figure 2. Infarct size (percentage of risk zone) of mice hearts. The isolated hearts of 5 different groups underwent ischemia/reperfusion protocols. The left hindlimb of anesthetized mice were subjected to remote ischemic conditioning (RIC) at cold (cold-RIC, $\mathrm{n}=11$ ) or warm (warm-RIC, $n=11$ ) temperatures or exposed to cold (cold-CTR, $n=11$ ) or warm (warm-CTR, $n=11$ ) temperatures only. In a group of warm-RIC mice, LY294002 (5 $\mu \mathrm{M}$; warm-RIC LY, $\mathrm{n}=7$ ) was infused into isolated hearts before the induction of ischemia. In (A) the first four groups are reported. In (B), warm-CTR and warm-RIC are reported again and placed next to the warm-RIC LY group to better highlight the statistical differences between these three groups $\left({ }^{* * *} p<0.0001{ }^{* * *} p<0.001\right)$.

\subsection{Warm-RIC Acted via the PI3K/Akt Pathway}

To evaluate the potential involvement of the PI3K/Akt pathway as a protective pathway activated in response to RIC, additional warm-RIC hearts were subject to IR in the presence of the PI3K inhibitor LY294002 (5 $\mu \mathrm{M})$. The administration of the inhibitor abolished the warm-RIC-induced protection. Indeed, after the LY294002 and IR protocol, the infarct size was $54 \pm 1.69 \%$ of risk zone ( $p=0.0002$ compared to the warm-RIC; Figure $2 \mathrm{~B}$ ).

Western blot analysis was performed on hearts harvested from warm groups treated, or not treated, with LY294002 to investigate Akt phosphorylation. The analysis showed an increase in warm-CTR and warm-RIC in the phosphorylation of Akt and a clear reduction in the phosphorylation of Akt in the presence of LY294002 (Figure 3). 


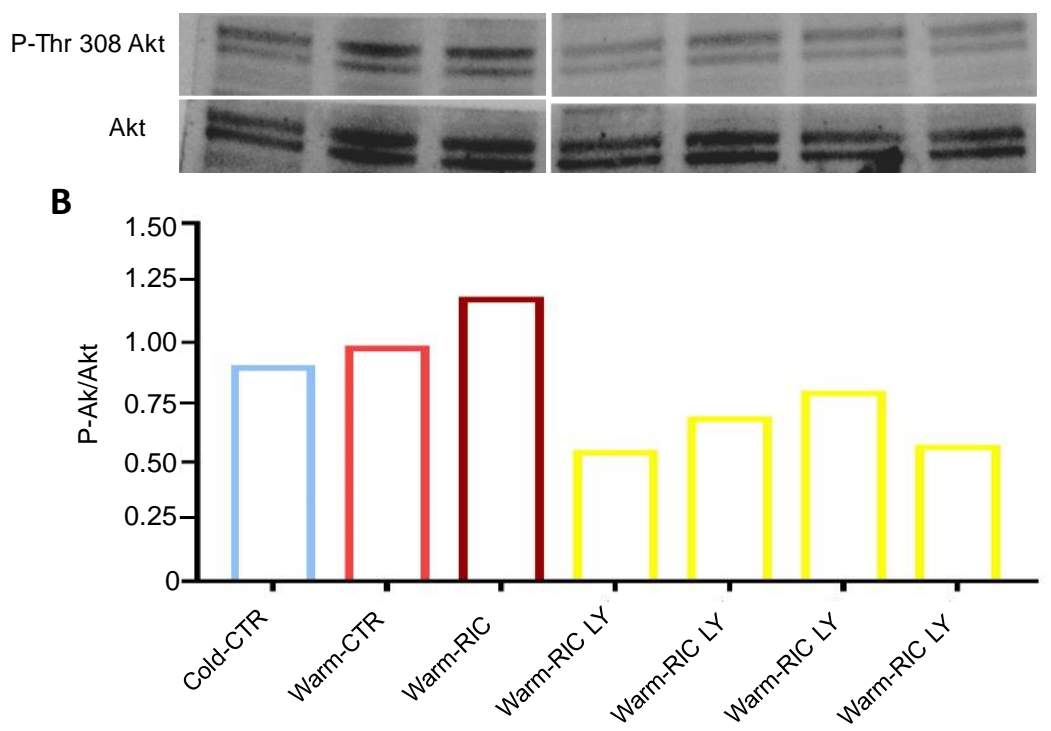

Figure 3. Western blot bands (A) and quantifications of phospho-Akt/Akt ratio (B) showing the effects of Akt phosphorylation inhibition following treatment with LY294002 in warm-RIC hearts compared to untreated warm-RIC and warm-CTR. Cold-CTR hearts are used as a reference group.

\section{Discussion}

Here we show that limb temperature influences the efficacy of RIC. Indeed, RIC performed at room temperature $\left(20^{\circ} \mathrm{C}\right)$, namely cold-RIC, induces a small non-consistent reduction of infarct size. However, RIC performed at warmer $\left(40^{\circ} \mathrm{C}\right) \operatorname{limb}$ temperature, namely warm-RIC, induces more consistent and significant cardioprotective effects, as revealed by a significantly reduced infarct size. Results suggest that inhibition of PI3K activity reduces the cardioprotective properties of warm-RIC. This latter effect is in agreement with studies reporting a causal involvement of PI3K/Akt activation in the at-risk cardiac area via cardioprotective maneuvers in rodents [2-4]. We propose that warm-RIC is a stronger stimulus and may be considered a promising approach that deserves to be tested in a clinical scenario.

We started from the consideration that in the ischemic muscle, such as in RIC, metabolic changes occur that are very similar to those that occur in a muscle that is working, such as in physical exercise. In both cases, for example, the levels of ADP, AMP, Pi and reactive oxygen species (ROS) increase, and glycogen and $\mathrm{pH}$ levels decrease [17]. Both RIC and exercise can trigger beneficial mechanisms for the whole organism [18]. However, a substantial difference between muscle undergoing either ischemic preconditioning or exercise is that in the first case the muscle cools, while in the second case the muscle warms up. Therefore, we decided to compare RIC procedures performed at two different temperatures of the limb subjected to intermittent ischemia. We choose to compare a typical room temperature $\left(20^{\circ} \mathrm{C}\right)$ with the typical temperature that muscles reach during exercise $\left(40^{\circ} \mathrm{C}\right)$.

To our knowledge, nobody has ever taken the temperature of the limb into account in RIC studies. Since both RIC and exercise can trigger beneficial mechanisms for the whole organism, we hypothesized that heating the limbs while doing RIC maneuvers could enhance the protective efficacy of the conditioning protocol, making it more similar to physical exercise.

It is well known that the development of infarct size may be temperature dependent. In particular, lower core body or heart temperature may reduce infarct size after IR protocols. Some authors have also studied whether lowering core temperature may affect the cardioprotection attained by RIC [19]. In the study by Verdouw et al., RIC and infarct size experiments were performed at $36-37$ or $30-31^{\circ} \mathrm{C}$. Pre-RIC was induced by a $15 \mathrm{~min}$ mesenteric artery occlusion or by a $15 \mathrm{~min}$ renal artery occlusion: both protocols 
were protective at lower core temperatures [19]. However, in this study, the myocardial IR protocol was conducted at a low temperature.

Temperature is among one of the most important variables to be controlled during ischemia/reperfusion studies, as it can greatly influence cell death and subsequent inflammatory processes. In vivo, in open chest acute experiments, the ischemic part will change temperature in an uncontrolled manner-depending mainly on room temperature, regardless of the use of a temperature-controlled operating table. Instead, in ex vivo Langendorff studies, the whole heart-including the ischemic part-is set to a well-controlled temperature. This avoids influences of temperature on infarct size [16]. Indeed, to avoid any influence of temperature directly on the myocardial IRI, we performed the pre-RIC in vivo and IR experiments after heart isolation, in an ex vivo model in which we can strictly control the heart temperature, which was set in all cases to $37^{\circ} \mathrm{C}$. Therefore, the infarct size reduction may not be attributed to a direct influence of temperature on IR injury, but to a potentiated mechanism starting from the ischemic limb, in which temperature plays a pivotal role.

The SAFE pathway, including the signal transducer and transcription activator (STAT)3 and STAT5, and the RISK pathway, including PI3K/Akt, can play a central role in RIC cardioprotection - in both humans and mice [20]. Indeed, in STAT5 knockout mice it was found that RIC-induced infarct size reduction occurs via the PI3K/Akt pathway and the anti-apoptotic cascade, thus confirming the importance of RISK in rodents [21]. These aspects make mice an appropriate animal species for studying RIC. Here, we confirm the importance of the PI3K/Akt survival pathway in warm-RIC cardioprotection, using a specific PI3K inhibitor. Indeed, WB analysis confirms Akt phosphorylation by warm-RIC, which is clearly blunted by the PI3K inhibitor (Figure 3).

Under our experimental conditions for all groups, only the leg was heated in vivo, and the entire heart was kept at identical temperatures during the ex vivo ischemia/reperfusion protocol. Therefore, it is unlikely that temperature played a direct role in the activation of the PI3K/Akt pathway. However, it is possible that one or more endogenous factors (more than 100 different signaling molecules may be released and involved in RIC [4,22]) are released from the warm-conditioned leg in greater quantities, reaching the threshold needed to activate the PI3K/Akt pathway in the myocardium. Future studies may ascertain this hypothesis.

RIC can be performed before ischemic events in programmed PTCA or cardio surgery intervention - that is, remote preconditioning - but it can be also used during an acute infarction (remote preconditioning) or after myocardial reperfusion (remote postconditioning) [2-4]. Here, we demonstrate the efficacy of warm-RIC for preconditioning. Nevertheless, we cannot rule out its superiority in preconditioning and postconditioning conditions. Additionally, these conditions may deserve to be tested in the future.

\section{Conclusions}

Warm-RIC obtained with transient ischemia of the hind-limb kept at $40{ }^{\circ} \mathrm{C}$ is more effective than cold-RIC (hind-limb kept at $20^{\circ} \mathrm{C}$ ) in reducing infarct size. Here, using a specific inhibitor for the PI3K/Akt pathway, we also confirmed that cardioprotective signal transduction causally involves an activation of Akt in the at-risk area of the mouse heart.

Changing the whole-body temperature is an approach proposed to reduce IRI [23-27]. However, it is a challenging task. Yet, to change only limb temperature in humans can be easily achieved. For instance, a simple thermal blanket around the ischemic limb while performing RIC may be an easy approach for testing in humans. Therefore, our observation that warm-RIC is more effective than room temperature RIC (cold-RIC) is a promising result that deserves to be tested in a clinical scenario, as it is simple and safe.

Author Contributions: Conceptualization, P.P., C.P. and M.B.; methodology, S.C., S.F., F.T. and M.S.; formal analysis, P.P., C.P., S.F. and M.S.; investigation, C.P., S.F., F.T. and M.S.; resources, P.P., C.P. and M.B.; data curation, P.P., C.P., S.F. and M.S.; writing-original draft preparation, P.P., C.P. and M.B.; writing-review and editing, P.P., C.P., M.B. and M.S.; supervision, P.P. and M.B.; project 
administration, P.P., C.P. and M.B.; funding acquisition, P.P. and C.P. All authors have read and agreed to the published version of the manuscript.

Funding: This research was funded by ex $60 \%-$ RILO (MUR) to P.P. and C.P.

Institutional Review Board Statement: The study was conducted according to the guidelines of the Declaration of Helsinki, and approved by the Institutional "Animal Use and Care Committee" of the University of Turin (protocol no: E669C.44).

Informed Consent Statement: Not applicable.

Data Availability Statement: Data are available at request from pasquale.pagliaro@unito.it; claudia.penna@unito.it; mara.brancaccio@unito.it.

Acknowledgments: The authors are grateful to Cecilia Thairi for thetechnical assistance. We thank Beneficenza San Paolo (B/2021/0159) for supporting P.P.

Conflicts of Interest: The authors declare no conflict of interest. The funders had no role in the design of the study; in the collection, analyses, or interpretation of data; in the writing of the manuscript, or in the decision to publish the results. All authors have read the journal's policy on the disclosure of potential conflicts of interest. Moreover, all authors declare that there are no conflicts of interest to disclose. All authors have read the journal's authorship statement.

\section{References}

1. Khan, M.A.; Hashim, M.J.; Mustafa, H.; Baniyas, M.Y.; Al Suwaidi, S.K.B.M.; AlKatheeri, R.; Alblooshi, F.M.K.; Almatrooshi, M.E.A.H.; Alzaabi, M.E.H.; Al Darmaki, R.S.; et al. Global Epidemiology of Ischemic Heart Disease: Results from the Global Burden of Disease Study. Cureus 2020, 12, e9349. [CrossRef]

2. Penna, C.; Granata, R.; Tocchetti, C.G.; Gallo, M.P.; Alloatti, G.; Pagliaro, P. Endogenous Cardioprotective Agents: Role in Pre and Postconditioning. Curr. Drug. Targets 2015, 16, 843-867. [CrossRef]

3. Hausenloy, D.J.; Yellon, D.M. Ischaemic conditioning and reperfusion injury. Nat. Rev. Cardiol. 2016, 13, 193-209. [CrossRef]

4. Kleinbongard, P.; Skyschally, A.; Heusch, G. Cardioprotection by remote ischemic conditioning and its signal transduction. Pflugers Arch. 2017, 469, 159-181, Erratum in Pflugers Arch. 2017, 469, 843. [CrossRef] [PubMed]

5. Cahill, T.J.; Kharbanda, R.K. Heart failure after myocardial infarction in the era of primary percutaneous coronary intervention: Mechanisms, incidence and identification of patients at risk. World J. Cardiol. 2017, 9, 407-415. [CrossRef] [PubMed]

6. Hausenloy, D.J.; Yellon, D.M. Myocardial ischemia-reperfusion injury: A neglected therapeutic target. J. Clin. Investig. 2013, 123, 92-100. [CrossRef]

7. Li, X.; Liu, M.; Sun, R.; Zeng, Y.; Chen, S.; Zhang, P. Protective approaches against myocardial ischemia reperfusion injury. Exp. Ther. Med. 2016, 12, 3823-3829. [CrossRef]

8. Ludman, A.J.; Yellon, D.M.; Hausenloy, D.J. Cardiac preconditioning for ischaemia: Lost in translation. Dis. Model Mech. 2010, 3, 35-38. [CrossRef]

9. Hausenloy, D.J.; Kharbanda, R.K.; Møller, U.K.; Ramlall, M.; Aarøe, J.; Butler, R.; Bulluck, H.; Clayton, T.; Dana, A.; Dodd, M.; et al. CONDI-2/ERIC-PPCI Investigators. Effect of remote ischaemic conditioning on clinical outcomes in patients with acute myocardial infarction (CONDI-2/ERIC-PPCI): A single-blind randomised controlled trial. Lancet (Lond. Engl.) 2019, 394, 1415-1424. [CrossRef]

10. Bromage, D.I.; Pickard, J.M.; Rossello, X.; Ziff, O.J.; Burke, N.; Yellon, D.M.; Davidson, S.M. Remote ischaemic conditioning reduces infarct size in animal in vivo models of ischaemia-reperfusion injury: A systematic review and meta-analysis. Cardiovasc. Res. 2017, 113, 288-297. [CrossRef] [PubMed]

11. Lecour, S.; Andreadou, I.; Bøtker, H.E.; Davidson, S.M.; Heusch, G.; Ruiz-Meana, M.; Schulz, R.; Zuurbier, C.J.; Ferdinandy, P.; Hausenloy, D.J.; et al. IMproving Preclinical Assessment of Cardioprotective Therapies (IMPACT) criteria: Guidelines of the EU-CARDIOPROTECTION COST Action. Basic Res. Cardiol. 2021, 116, 52. [CrossRef] [PubMed]

12. Pickard, J.M.; Davidson, S.M.; Hausenloy, D.J.; Yellon, D.M. Co-dependence of the neural and humoral pathways in the mechanism of remote ischemic conditioning. Basic Res. Cardiol. 2016, 111, 50. [CrossRef]

13. Sbroggiò, M.; Bertero, A.; Velasco, S.; Fusella, F.; De Blasio, E.; Bahou, W.F.; Silengo, L.; Turco, E.; Brancaccio, M.; Tarone, G. ERK1/2 activation in heart is controlled by melusin, focal adhesion kinase and the scaffold protein IQGAP1. J. Cell. Sci. 2011, 124, 3515-3524. [CrossRef] [PubMed]

14. Penna, C.; Brancaccio, M.; Tullio, F.; Rubinetto, C.; Perrelli, M.G.; Angotti, C.; Pagliaro, P.; Tarone, G. Overexpression of the muscle-specific protein, melusin, protects from cardiac ischemia/reperfusion injury. Basic Res. Cardiol. 2014, 109, 418. [CrossRef] [PubMed]

15. Chaudhary, K.R.; Batchu, S.N.; Das, D.; Suresh, M.R.; Falck, J.R.; Graves, J.P.; Zeldin, D.C.; Seubert, J.M. Role of B-type natriuretic peptide in epoxyeicosatrienoic acid-mediated improved post-ischaemic recovery of heart contractile function. Cardiovasc. Res. 2009, 83, 362-370. [CrossRef] 
16. Bøtker, H.E.; Hausenloy, D.; Andreadou, I.; Antonucci, S.; Boengler, K.; Davidson, S.M.; Deshwal, S.; Devaux, Y.; Di Lisa, F.; Di Sante, M.; et al. Practical guidelines for rigor and reproducibility in preclinical and clinical studies on cardioprotection. Basic Res. Cardiol. 2018, 113, 39. [CrossRef]

17. Allen, D.G.; Lamb, G.D.; Westerblad, H. Skeletal muscle fatigue: Cellular mechanisms. Physiol. Rev. 2008, 88, 287-332. [CrossRef] [PubMed]

18. Penna, C.; Alloatti, G.; Crisafulli, A. Mechanisms Involved in Cardioprotection Induced by Physical Exercise. Antioxid. Redox Signal. 2020, 32, 1115-1134. [CrossRef]

19. Verdouw, P.D.; Gho, B.C.; Koning, M.M.; Schoemaker, R.G.; Duncker, D.J. Cardioprotection by ischemic and nonischemic myocardial stress and ischemia in remote organs. Implications for the concept of ischemic preconditioning. Ann. N. Y. Acad. Sci. 1996, 793, 27-42. [CrossRef]

20. Comità, S.; Femmino, S.; Thairi, C.; Alloatti, G.; Boengler, K.; Pagliaro, P.; Penna, C. Regulation of STAT3 and its role in cardioprotection by conditioning: Focus on non-genomic roles targeting mitochondrial function. Basic Res. Cardiol. 2021, 116, 56. [CrossRef]

21. Chen, H.; Jing, X.Y.; Shen, Y.J.; Wang, T.L.; Ou, C.; Lu, S.F.; Cai, Y.; Li, Q.; Chen, X.; Ding, Y.J.; et al. Stat5-dependent cardioprotection in late remote ischaemia preconditioning. Cardiovasc. Res. 2018, 114, 679-689. [CrossRef] [PubMed]

22. Heusch, G. Molecular basis of cardioprotection: Signal transduction in ischemic pre-, post-, and remote conditioning. Circ. Res. 2015, 116, 674-699. [CrossRef]

23. Guo, Y.; Flaherty, M.P.; Wu, W.J.; Tan, W.; Zhu, X.; Li, Q.; Bolli, R. Genetic background, gender, age, body temperature, and arterial blood $\mathrm{pH}$ have a major impact on myocardial infarct size in the mouse and need to be carefully measured and/or taken into account: Results of a comprehensive analysis of determinants of infarct size in 1074 mice. Basic Res. Cardiol. 2012, 107, 288. [CrossRef]

24. Chien, G.L.; Wolff, R.A.; Davis, R.F.; van Winkle, D.M. "Normothermic range" temperature affects myocardial infarct size. Cardiovasc. Res. 1994, 28, 1014-1017. [CrossRef] [PubMed]

25. Hale, S.L.; Kloner, R.A. Myocardial temperature in acute myocardial infarction: Protection with mild regional hypothermia. Am. J. Physiol. 1997, 273, H220-H227. [CrossRef]

26. Schwartz, L.M.; Verbinski, S.G.; Vander Heide, R.S.; Reimer, K.A. Epicardial temperature is a major predictor of myocardial infarct size in dogs. J. Mol. Cell. Cardiol. 1997, 29, 1577-1583. [CrossRef]

27. Pagliaro, P.; Aragno, M.; Penna, C. Role of temperature in myocardial ischemic injury and protection by conditioning. Cond. Med. 2020, 3, 31-46. 